\title{
Erratum: Nuclear electric dipole moments in chiral effective field theory
}

J. Bsaisou, ${ }^{a}$ J. de Vries, ${ }^{a}$ C. Hanhart, ${ }^{a, b}$ S. Liebig, ${ }^{a}$ Ulf-G. Meißner, ${ }^{a, b, c, d}$ D. Minossi, ${ }^{a}$ A. Nogga ${ }^{a, b}$ and A. Wirzba ${ }^{a, b}$

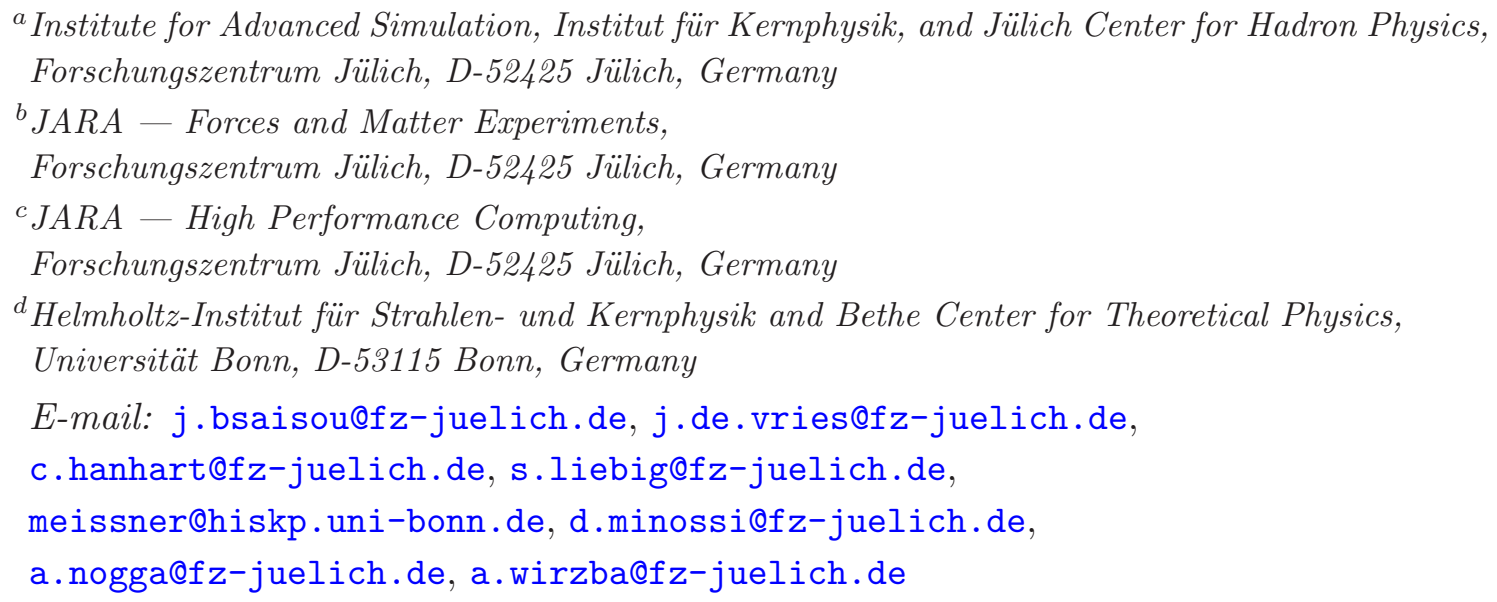

ERRATUM TO: JHEP03(2015)104

ARXIV EPRINT: 1411.5804

As first observed in ref. [1], the usual weight factors of the neutron $\left(d_{n}\right)$ and proton $\left(d_{p}\right)$ single-nucleon contribution to the electric dipole moment of the deuteron are lacking a small wave-function-dependent term resulting from the subleading ${ }^{3} D_{1}$ component of the deuteron wave function. A simple calculation reveals that the total single-nucleon contribution is

$$
d_{2} \mathrm{H}, \text { single }=\left(1-\frac{3}{2} P_{D}\right)\left(d_{n}+d_{p}\right),
$$

where $P_{D}$ is the probability of the deuteron ${ }^{3} D_{1}$-state, which of course depends on the choice of the wave function.

Therefore the values 1.00 of the $d_{n}$ and $d_{p}$ weight factors have to be modified in the following places of our paper [2]:

(i) in the first two rows of table 1 , see the enclosed table $1^{\prime}$, 


\begin{tabular}{|c|r|r|r|c|}
\hline label & $\mathrm{N}^{2} \mathrm{LO} \chi \mathrm{EFT}$ & $\mathrm{A} v_{18}$ & $\mathrm{CD}-\mathrm{Bonn}$ & units \\
\hline$d_{n}$ & $0.939 \pm 0.009$ & 0.914 & 0.927 & $d_{n}$ \\
$d_{p}$ & $0.939 \pm 0.009$ & 0.914 & 0.927 & $d_{p}$ \\
$g_{1}$ & $-0.183 \pm 0.017$ & -0.186 & -0.186 & $g_{1} e \mathrm{fm}$ \\
$\Delta f_{g_{1}}$ & $0.748 \pm 0.138$ & 0.703 & 0.719 & $\Delta e \mathrm{fm}$ \\
\hline
\end{tabular}

Table $\mathbf{1}^{\prime}$. The new entries of table 1 . Captions as in table 1 . Note that the new $d_{n}$ and $d_{p}$ weight factors displayed for the $\mathrm{A} v_{18}$ potential exactly agree with the ones of ref. [1].

(ii) in the first bracket on the right-hand side of eq. (3.2),

$$
d_{2 \mathrm{H}}=(0.939 \pm 0.009)\left(d_{n}+d_{p}\right)-\left[(0.183 \pm 0.017) g_{1}-(0.748 \pm 0.138) \Delta\right] e \mathrm{fm},
$$

(iii) implicitly in the first bracket of eq. (4.9),

$$
\begin{aligned}
d_{2 \mathrm{H}}^{\theta}=\bar{\theta} \cdot\{[(0.56 \pm 0.01 \pm 1.59)] & \\
& \quad-(0.62 \pm 0.06 \pm 0.28)-(0.28 \pm 0.05 \pm 0.07)\} \cdot 10^{-16} e \mathrm{~cm}
\end{aligned}
$$

such that " $(0.6 \pm 1.7)$ " is replaced by "( $0.56 \pm 0.01 \pm 1.59)$ ", where the first uncertainty is the nuclear one, while the second is the hadronic one,

(iv) explicitly on the left-hand side of eq. (4.10),

$$
d_{2 \mathrm{H}}^{\theta}-0.94\left(d_{p}^{\theta}+d_{n}^{\theta}\right)=-\bar{\theta} \cdot(0.89 \pm 0.30) \cdot 10^{-16} e \mathrm{~cm},
$$

(v) and on the left-hand side of eq. (5.5),

$$
\begin{aligned}
d_{2 \mathrm{H}}^{L R}-0.94\left(d_{p}^{L R}+d_{n}^{L R}\right) & =\Delta^{L R}[(1.37 \pm 0.13 \pm 0.41)+(0.75 \pm 0.14) \pm 0.1] e \mathrm{fm} \\
& =\Delta^{L R}(2.1 \pm 0.5) e \mathrm{fm}
\end{aligned}
$$

In the latter two cases the uncertainty of the single-nucleon contributions can safely be neglected.

Open Access. This article is distributed under the terms of the Creative Commons Attribution License (CC-BY 4.0), which permits any use, distribution and reproduction in any medium, provided the original author(s) and source are credited.

\section{References}

[1] N. Yamanaka and E. Hiyama, Enhancement of the CP-odd effect in the nuclear electric dipole moment of ${ }^{6} \mathrm{Li}$, arXiv: 1503.04446 [INSPIRE].

[2] J. Bsaisou et al., Nuclear electric dipole moments in chiral effective field theory, JHEP 03 (2015) 104 [arXiv: 1411.5804] [INSPIRE]. 